\title{
Pediatric Bithalamic Anaplastic Astrocytoma Followed by Distant Progression to Glioblastoma in the Region of Corpus Callosum
}

\author{
Nemanja Jovanovic ${ }^{1}$, Rosanda Ilic ${ }^{1}$, Aleksandra Paunovic ${ }^{1}$, Zarko Nedeljkovic ${ }^{1}$, Filip Milisavlje- \\ vic $^{1}$, Ivan Bogdanovic ${ }^{1}$, Igor Lazic ${ }^{3}$, Ivan Vukasinovic ${ }^{1 *}$, Vuk Aleksic ${ }^{2}$ and Danica Grujicic ${ }^{1}$ \\ ${ }^{1}$ Faculty of Medicine, University of Belgrade, Serbia
}

${ }^{2}$ Department of neurosurgery, Clinical Hospital Center Zemun, Belgrade, Serbia

${ }^{3}$ Center for anesthesiology, Clinical Center of Serbia, Serbia

*Corresponding author: Ivan Vukasinovic, Clinic of Neurosurgery, University Clinical Center of Serbia, Belgrade, Serbia. Koste Todorovica 4, 11000 Belgrade, Serbia.

To Cite This Article: Nemanja Jovanovic, Rosanda Ilic, Aleksandra Paunovic, Zarko Nedeljkovic, Ivan Vukasinovic, et al., Pediatric Bithalamic Anaplastic Astrocytoma Followed by Distant Progression to Glioblastoma in the Region of Corpus Callosum. Am J Biomed Sci \& Res. 2021 - 13(5). AJBSR.MS.ID.001909. DOI: 10.34297/AJBSR.2021.13.001909.

Received: 畊 June 04, 2021; Published: 制 July 29, 2021

\begin{abstract}
Primary bilateral thalamic tumors are extremely rare entity. We present a case of a 5-year-old child admitted with signs of raised intracranial pressure. Brain MRI showed huge bithalamic lesion with subsequent hydrocephalus. After ventriculo peritoneal shunt, direct operation was performed, and most of the right sided tumor mass was removed. Diagnosis was anaplastic astrocytoma. Although quarterly MRI did not show signs of tumor regrowth, twenty-one months after surgery child was referred to our department with acute consciousness deterioration, and hemorrhage in the region of the corpus callosum. After hematoma evacuation patient has recovered. Pathohistological examination showed glioblastoma, so after two weeks child was again operated, and gross tumor resection was performed. However, signs of leptomeningeal spread occurred, and patient died two months after the last operation.

In most cases bilateral thalamic tumors cause subsequent hydrocephalus that should be treated with ETV or VP shunt. Bilateral thalamic tumors are probably more often malignant than previously thought. Distant progression in corpus callosum seen in our patient confirms theory that bithalamic gliomas can be genetically like "butterfly" tumors.
\end{abstract}

Keywords: Bilateral Thalamic Tumors, Anaplastic Astrocytoma, Glioblastoma

\section{Introduction}

Pediatric thalamic tumors are rare, and their frequency is estimated between $0.84 \%$ to $5.2 \%$ of all intracranial tumors. Primary bilateral thalamic tumors are extremely rare [1]. Steinbok et al. [2] reported review of seventy-two thalamic tumors of which 10 patients had bilateral lesion. They are histologically benign, showing slow growth and reaching huge dimensions at the time of diagnosis, often causing CSF flow obstruction. Because of their proximity to important and deep brain structures and the risk of devastating postoperative morbidity they are seldom operated, and surgical treatment most often consider only management of hydrocephalus and tumor biopsy [2,3]. However, with better technology, surgical resection of some thalamic tumors has become achievable with acceptable morbidity.

\section{Case Report}

We present a case of a 5-year-old girl admitted with signs of raised Intracranial Pressure (headache, vomiting and lethargy) (ICP) and left sided hemiparesis that lasted for two weeks. Systemic examination was normal and neurological examination revealed upward gaze palsy, mild left sided hemiparesis, and chronic 
papilledema with 2.5D on the left, and 3.5D on the right eye. Brain CT scan showed large isodense mass involving bithalamic region with right sided predominance and ventricular enlargement. There was no contrast enhancement (Figure 1). Brain MRI showed huge bithalamic lesion, with some right sided predominance. It was uniformly isointense on T1 weighted and, hyperintense on T2 and FLAIR weighted images, with no contrast enhancement. Lateral ventricles were enlarged (Figure 2). After VP shunt placement and corticosteroid therapy symptoms resolved. In the second surgery patient was operated through the right, nondominant parietal lobulus since tumor mass was larger in the right thalamus, filling the body of the right lateral ventricle with significant lateral and posterior extension. During the operation body of the right ventricle was entered, and intraventricular tumor mass was removed. Tumor was without clear border to right thalamus. Most of the right sided mass was removed. Postoperative course went uneventful.

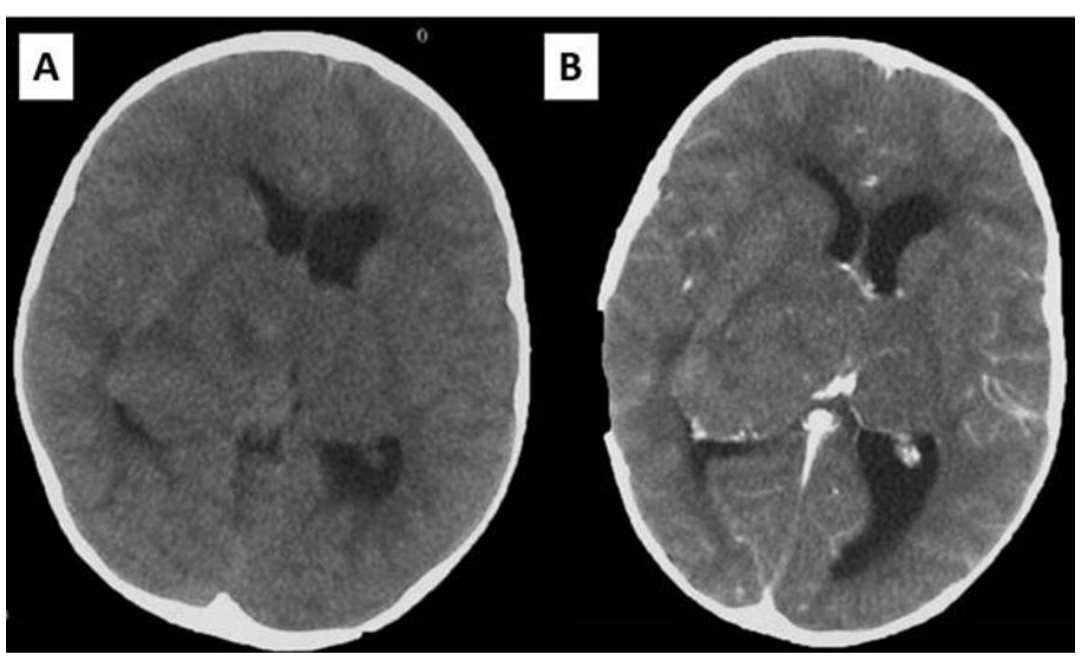

Figure 1: Initial brain CT showing the bithalamic tumor: (A) native study; (B) contrast enhanced study.

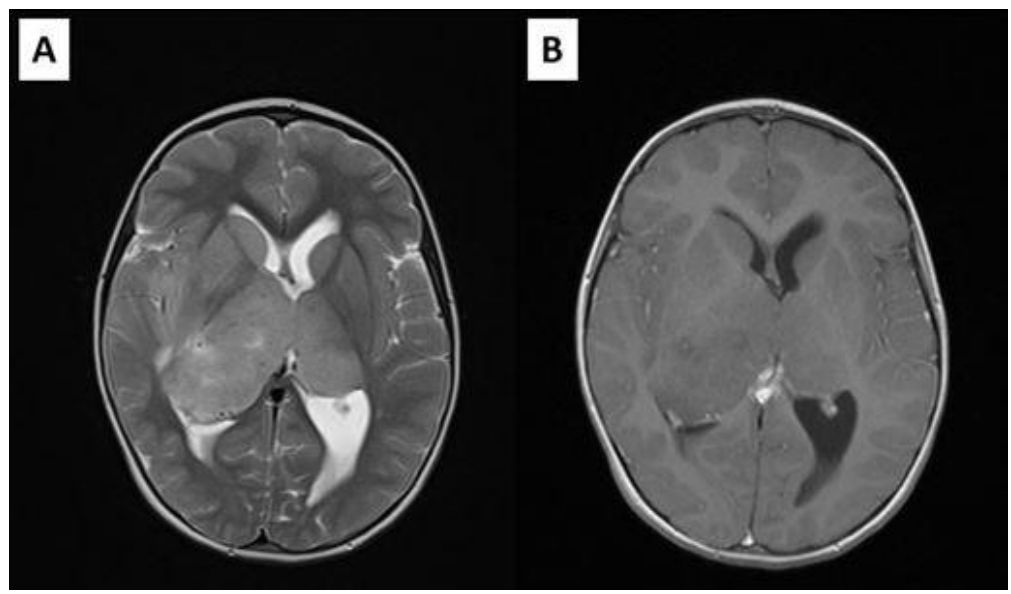

Figure 2: Preoperative MRI showing the bithalamic tumor: (A) T2W; (B) postcontrast T1 study.

Pathohistological examination showed in some parts of tumor histological picture of low-grade astrocytoma with entrapped neurons, and in the other parts cellular tumor with pronounced mitotic activity and nuclear atypia. There was no necrosis and microvascular proliferation. Tumor cell in both zones expressed GFAP, Vimentin, S-100 and INI1. Also, p53 positive nuclei were seen. Ki-67 positive nuclei were rare in the zone of low cellularity and very frequent in cellular zone (Ki-67 about 20\%). Definitive diagnosis was anaplastic astrocytoma.
Postoperative MRI showed residual tumor in the left thalamic region, and radiotherapy was performed, followed by chemotherapy. Residual tumor regression was confirmed with MRI, and the patient was stable almost for 2 years. She was monitored with MRI every 3 months (Figure 3). However, twenty-one months after surgery and two months after previous brain MRI which showed no signs of tumor regrowth, child was referred to our department with acute consciousness deterioration, drowsy, adynamic with Glasgow coma score of 10 points. Brain CT showed bleeding in the region of the 
corpus callosum, and MRI, confirmed bleeding within the genum of corpus callosum and symmetrical extension into the white matter of both frontal lobes. On the post contrast tomograms tumor component could not be identified (Figure 4). The patient had low platelet number, so impaired coagulation was suspected as the cause of bleeding. MSCT angiography was normal. Hematoma was evacuated and biopsy samples were taken. The child recovered well after operation. Pathohistological examination revealed that the cause of bleeding was high grade glial tumor. This time the definite diagnosis was Glioblastoma.

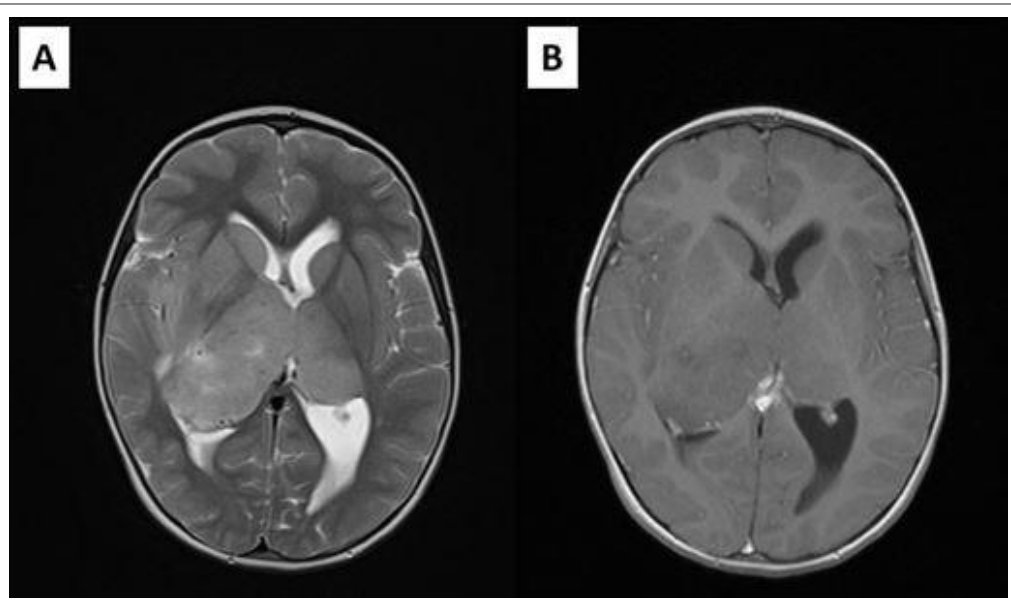

Figure 3: Brain MRI 18 months after operation: (A) post-contrast T1W; (B) T2W.

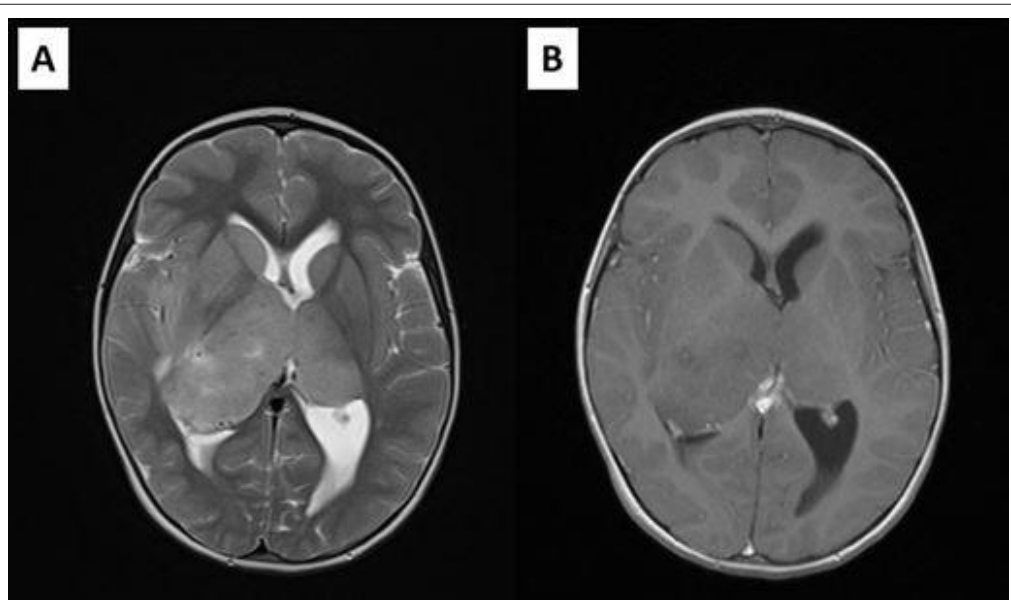

Figure 4: Imaging studies demonstrating hemorrhage: (A) Brain CT showing hemorrhage in the region of corpus callosum; (B) Brain MRI showing signs of bleeding within the corpus callosum and extension into the white matter of both frontal lobes presented in postcontrast T1W; (C) sagittal T1W sequence, (D) T2W sequence.

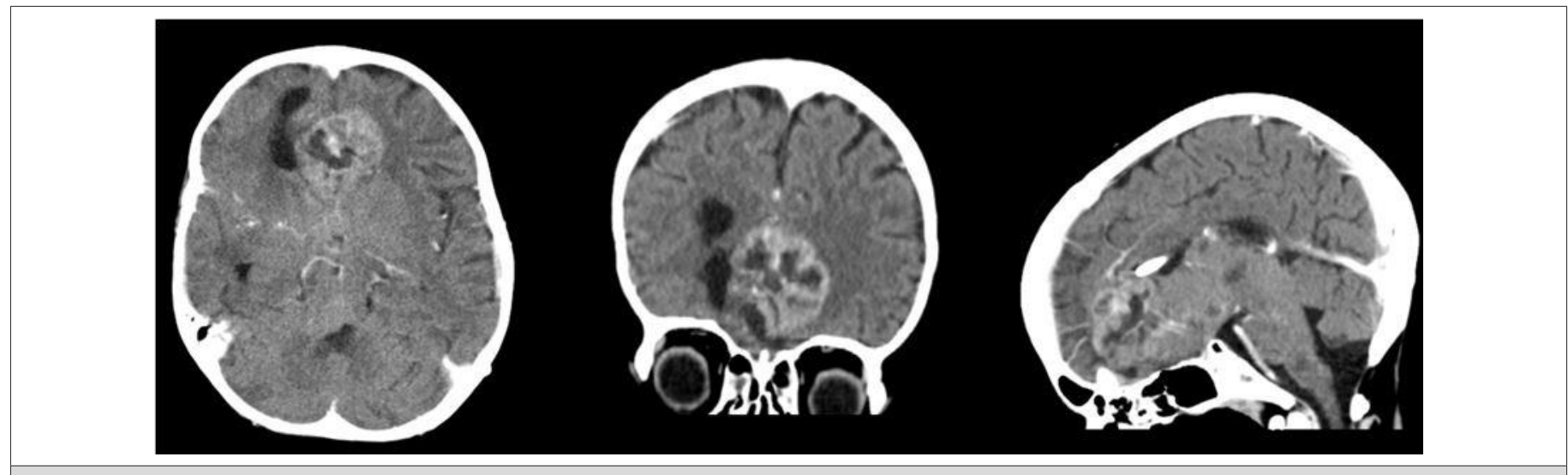

Figure 5: Brain CT showing tumor regrowth. 
The child was presented to the tumor board, with the decision to continue the treatment with chemotherapy. In the next two weeks the child developed drowsiness, fatigue and cough, difficulties with swallowing and chewing. Brain CT scan showed tumor regrowth in the region of corpus callosum, so we again decided to operate the child (Figure 5). After resection of visible part of tumor, we performed falx resection and removed the left sided part of the tumor. Although extent of tumor resection was significant and no damage to the eloquent regions was done, the patient did not achieve satisfactory recovery (Figure 6). Probable cause was the microscopic infiltration of the brain stem and leptomeningeal dissemination, which could not be visualized by the MRI. No further oncological therapy was conducted, and the child died two months after the last operation.

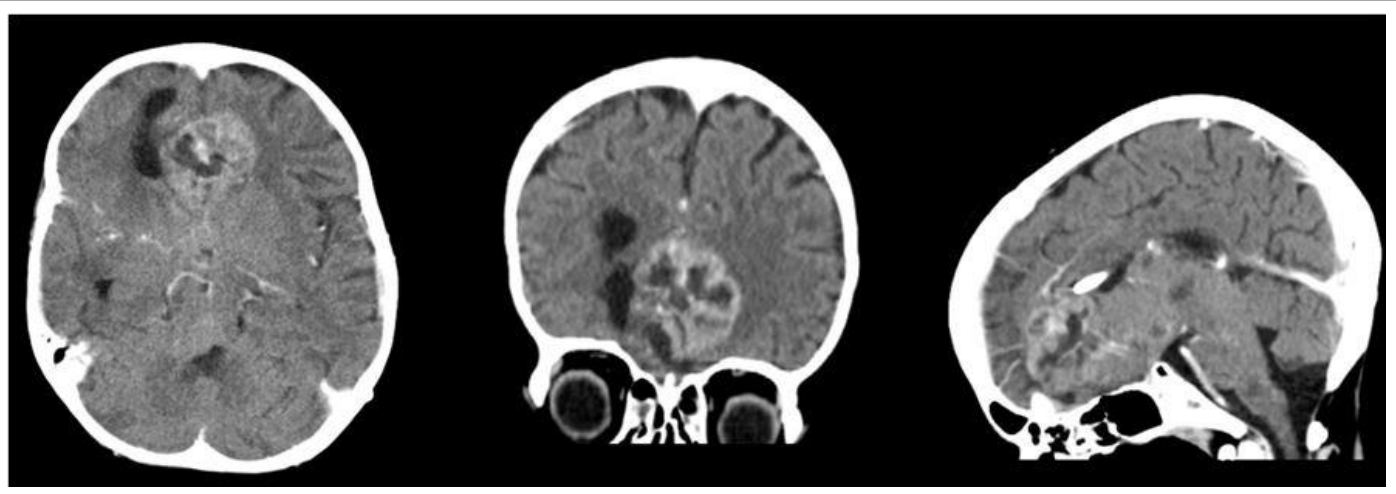

Figure 6: Brain CT following third surgery.

\section{Discussion}

Thalamic gliomas are rare tumors that make up about 1-5 \% of pediatric intracranial tumors. The bilateral thalamic tumors are sporadic and account for about $15 \%$ of tumors in thalamic region [2]. According to literature, the mean age at presentation is about 6 years, with relatively short mean duration of symptoms of about 2.5 month. Our patient's age was consistent with the patients presented in published series, with a very expeditious diagnosis within 2 weeks of symptom onset. Thalamic tumors are generally presented with signs and symptoms of increased ICP and motor deficits $[2,4]$, which was also case in our patient.

In the study of Steinbok, the majority (90\%) of bithalamic tumors progressed, with the mean time to progression being 14 months [2]. In our case, patient time to progression was about 21 months, and this can be explained with relatively fast diagnosis after symptoms onset, aggressive surgical debulking followed with combined therapy.

Hydrocephalus and ventriculomegaly seemed to be a sine qua non conditions with bithalamic lesions and in most cases required some form of CSF diversion due to raised ICP. Diagnostic biopsy and CSF derivation are often the only surgical procedures that can be performed in these patients for symptomatic relief and for pathological diagnosis [2]. Ventricular enlargement is usually confined to lateral ventricles, caused with the obstruction at the level of foramen Monroo, or posterior III ventricle, and sometimes may be asymmetrical [5,6]. As in other pediatric tumors there is debate about the optimal method of hydrocephalus management.
As radical surgery with definitive deopstruction of cerebrospinal fluid pathways cannot be achieved, temporary ventricular drainage is insufficient. Endoscopic approach can be considered if anterior part of III ventricle is not obstructed.

This is also safe and efficient way to provide biopsy specimen. Depending on the site of obstruction the patients with blocked communication between lateral ventricles, endoscopic pellucidotomy might be considered as the additional procedure to VP shunt. VP shunt is another method for hydrocephalus treatment [2]. We decided to place the VP shunt before main operation to reduce intracranial pressure, and there was an immediate improvement in the child's neurological condition. Also, we wanted to achieve as much as possible brain relaxation before the open surgery procedure. Open surgery is controversial question in bithalamic tumors. Prior studies suggest that these tumors are inoperable due to diffuse and bilateral extension, and no case of radical removal has been described so far [7]. Main role of surgery is to get tissue for histological diagnosis.

Also, tumor reduction can improve the oncological treatment. However, there is a lack of evidence that extent of resection influences overall survival in patients with bithalamic tumors, and due to high risk of additional morbidity and mortality in these tumors total resection should not be the goal. Different approaches are used for these tumors, such as infratentorial supracerebellar, interhemispheric transcallosal, or transsylvian approach. Biopsy can be performed endoscopically or under stereotactic guidance [7]. We decided to approach through the right superior parietal lobule, due to nondominant hemisphere localization and posterior 
and lateral tumor mass extension. We achieved significant tumor reduction, with residual tumor in the left thalamic region. Bithalamic tumors are showed to be less responsive to adjuvant chemo and radiotherapy in comparison to unithalamic lesion. The relative unresponsiveness to adjuvant therapy and the inability of gross tumor resection leads to a poor outcome $[2,7,8]$. We decided to conduct radiotherapy followed by chemotherapy that led to significant residual tumor regression. Aggressive treatment of new tumor failed due to leptomeningeal dissemination.

Previous studies showed that bithalamic tumors are predominantly low-grade astrocytomas. However, on the contrary to earlier assumption that these tumors are usually benign $[3,9]$, recent reviews of literature showed that they are malignant in more than half of the cases, with most frequent histological type being anaplastic astrocytoma $[2,6]$. Therefore, the frequency of malignant tumors might be underestimated. Needle and endoscopic biopsy, thatare usually used to establish diagnosis, provide small samples, so areas of increased mitotic activity, necrosis and neovascularization might be missed. In our patient, large specimen showed two distinct zones, one resembling low grade glioma, and other having features of high-grade glioma. This, along with specific molecular pattern might explain poor prognosis and unresponsiveness to the treatment $[3,5]$.

After WHO classification from 2016, when diffuse midline glioma, H3 K27M-mutant was introduced as the new entity, specific for pediatric patients with midline gliomas (diffuse intrinsic pontine gliomas, thalamic gliomas) and poor prognosis, it could have been expected that bithalamic gliomas will also have this genetic pattern, but recent investigations excluded this possibility $[5,10]$. This confirms hypothesis of Di Rocco et al, that bithalamic pediatric gliomas are a distinct entity [3]. Distant tumor that appeared in our patient in the region of corpus callosum (also localized in the midline) in the absence of local progression in thalamic region might present dissemination along the white matter fibers, or new entity. Certainly, this could lead to hypothesis that bithalamic gliomas might be genetically similar not to pontine gliomas, but to gliomas of corpus callosum, so called butterfly tumors.

\section{Conclusion}

Primary bilateral thalamic gliomas are rare tumors difficult to treat. They are more often malignant, and the real frequency of high-grade tumors might be underestimated. If present, hydrocephalus should be treated with ETV or VP shunt. The role of tumor debulking is not clear. We showed that good local control can be achieved with combined aggressive surgical and oncological treatment. Distant progression in corpus callosum could suggest that bithalamic gliomas are genetically like "butterfly" tumors.

\section{Conflict of Interests}

The authors declare no conflict of interests.

\section{References}

1. Yoshida M, Fushiki S, Takeuchi Y, Imamura T, Shigata T, et al. (1998) Diffuse bilateral thalamic astrocytomas as examined serially by MRI. Childs NervSyst 14(8): 384-388.

2. Steinbok P, Gopalakrishnan CV, Hengel AR, Vitali AM, Poskitt K, et al. (2016) Pediatric thalamic tumors in the MRI era: a Canadian perspective. Childs NervSyst 32: 269-280.

3. Di Rocco C, Iannelli A (2002) Bilateral thalamic tumors in children. Childs NervSyst 18: 440-444.

4. Cuccia V, Monges J (1997) Thalamic tumors in children. Childs NervSyst 13: 514-520.

5. Broniscer A, Hwang SN, Chamdine O, Lin T, Pounds S, et al. (2018) Bithalamicgliomas may be molecularly distinct from their unilateral high-grade counterparts. Brain Pathol 28(1): 112-120.

6. Niu X, Wang T, Yang Y, Gan Y, Li J, et al. (2018) Prognostic Factors for the Survival Outcome of Bilateral Thalamic Glioma: An Integrated Survival Analysis. World Neurosurg 110: 222-230.

7. Rajput DK, Mehrotra A, Srivastav AK, Kumar R, Mahapatra AK (2010) Bilateral thalamic glioma in a 6-year-old child. J Pediatr Neurosci 5(1): 45-48.

8. Fernandez C, Maues de Paula A, Colin C, Quilichini B, Bouvier Labit C, et al. (2006) Thalamic gliomas in children: an extensive clinical, neuroradiological and pathological study of 14 cases. Childs NervSyst 22: $1603-1610$

9. Puget S, Crimmins DW, Garnett MR, Grill J, Oliveira R, et al. (2007) Thalamic tumors in children: a reappraisal. J Neurosurg 106(5): 354362.

10. Louis DN, Perry A, Reifenberger G, von Deimling A, Figarella Branger D, et al. (2016) The 2016 World Health Organization Classification of Tumors of the Central Nervous System: a summary. Acta Neuropathol 131(6): 803-820. 